\section{La vie sans cavéoles}

Laurence Abrami, F. Gisou van der Goot, Frank Lafont résultat est remarquable parce qu'il montre que l'inactivation d'un seul gène
Département de Biochimie, Université de Genève, 30 quai Ernest-Ansermet, $\mathrm{CH}-1211$ Genève 4, Suisse. peut induire la suppression d'un organite. Jamais un organite entier n'avait été supprimé jusqu'à ce jour. Curieusement, ces souris vivent et sont ferment le rôle de la cavéoline, l'établissement de souris transgéniques avec inactivation du gène de la cavéoline-l était un événement très attendu. Deux études viennent de répondre à cette attente [3, 4].

L'étude de ces souris transgéniques montre que l'absence de cavéoline- 1 n'a aucun effet sur le niveau d'expression de la cavéoline-3 mais curieusement entraîne une réduction de $95 \%$ ou plus du niveau d'expression de la cavéoline-2, les $5 \%$ restant n'étant pas à la membrane plasmique mais dans un compartiment péri-nucléaire. Les molécules de cavéoline-2, ne pouvant plus former d'hétéro-oligomères avec la cavéoline-1, seraient dégradées par les protéasomes comme le suggère l'emploi d'un inhibiteur de ce complexe. Plus intéressante est l'analyse morphologique des cellules de ces souris transgéniques qui montre qu'elles n'ont plus de cavéoles à leur membrane plasmique. Ce tiles montrant que la vie existe sans cavéoles. De façon surprenante, la composition des lipoprotéines sanguines et le taux de cholestérol dans les HDL restent normaux dans les souris mutantes thèse émise dans les années 1950, les cavéoles ne sont pas indispensables à la transcytose.

L'absence de cavéoline-l n'est cependant pas sans effet. L'examen histologique des poumons a permis d'observer une anomalie sévère de la structure tissulaire avec une épaisseur accrue des septums alvéolaires. Cet épaississement est dû à la présence de dépôts fibreux extracellulaires et à une hypertrophie des pneumocytes de type II mais surtout à une prolifération de cellules endothéliales. Cette hyperprolifération pourrait être à l'origine du manque de résistance à l'effort des souris mutantes. suggérant que, contrairement à l'hypo- lumière des vaisseaux vers les tissus voisins par «transcytose». Depuis, de nouveaux rôles lui ont été attribués aussi bien dans le transport intracellulaire du cholestérol, que dans l'activation de voies de transduction du signal. De plus, le gène de la cavéoline-l étant localisé à proximité d'un locus que l'on présume important dans la suppression de tumeurs, des mutations de ce gène pourraient êtres impliquées dans certains cancers. Afin de mieux comprendre la fonction des

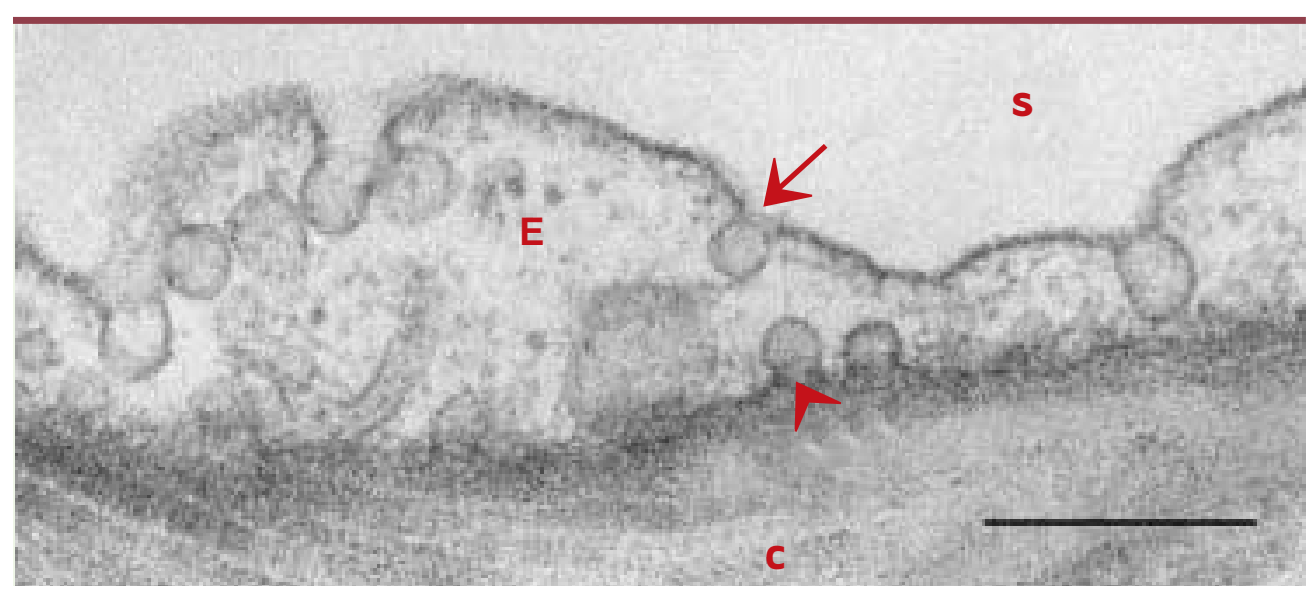

Figure 1. Cellule endothéliale pulmonaire reposant à sa partie basale sur du tissu conjonctif, visualisée en microscopie électronique à transmission. S: sang; $\varepsilon$ : cellule endothéliale; $C$ : tissu conjonctif. La flèche pointe une cavéole s'ouvrant vers la lumière endothéliale et la tête de flèche vers une cavéole s'ouvrant vers le tissus conjonctif. Barre: $250 \mathrm{~nm}$. Image reproduite avec la permission du Dr Paul Verkade, MPI-CBG (Dresde, Allemagne). 
Ces études indiquent qu'il existe donc effectivement un lien entre l'expression de la cavéoline-l et le contrôle de la division cellulaire comme le suggéraient d'anciens travaux rapportant l'inhibition de la prolifération de cellules tumorales après sur-expression de cavéoline-1. Cette hyper-prolifération semble indépendante de la cascade de signalisation de la p 42/44 MAP-kinase. Il est intéressant de noter qu'aucune tumeur précoce spontanée n'est apparue dans les souris mutantes.

Les souris transgéniques ont aussi des dysfonctionnements du système vasculaire, dus probablement à une hyperactivation de la synthase endothéliale du monoxyde d'azote (eNOS). La cavéoline-1 agit effectivement comme un inhibiteur de eNOS. II est possible qu'un des rôle des cavéoles soit d'inhiber, par séquestration dans l'organite, toute une série de molécules impliquées dans la signalisation. Une de ces molécules pourrait être la NOS inductible comme le suggère le fait que les souris transgéniques mâles soient en érection permanente [5].

Comment expliquer les effets relativement modérés observés après suppression complète d'un organite potentiellement impliqué dans des processus cellulaires aussi importants que le trafic membranaire, la signalisation et la suppression de tumeurs? Des travaux antérieurs ont montré que la suppression de gènes inhibant le cycle cellulaire n'entraîne pas nécessairement la formation de tumeurs. Par ailleurs, on ne peut exclure que d'autres molécules compensent la perte d'expression de la cavéoline-1. Celles-ci ne seraient pas exclusivement des protéines mais aussi des lipides. Effectivement, les cavéoles constituent des micro-domaines à la membrane plasmique non seulement par le fait qu'elles soient invaginées et contiennent des cavéolines, mais aussi par leur composition lipidique particulière, caractéristique de ce qui a été désigné sous le terme de «radeaux lipidiques» [1]. L'hypothèse de ces radeaux propose que dans les membranes contenant suffisamment de cholestérol et de sphingolipides, deux phases fluides coexistent: une phase désordonnée enrichie en phospholipides et une ordonnée, les radeaux, enrichie en sphingolipides et cholestérol. Ces radeaux sont de petite taille $(<100 \mathrm{~nm})$. Les cavéoles seraient le résultat d'une coalescence de certains radeaux obtenus par oligomérisation des cavéolines-1 et-2. Au cours d'une conférence EURESCO récemment consacrée aux microdomaines, radeaux lipidiques, et cavéoles [2], il est clairement apparu qu'en fait ces radeaux sont certainement modulables et capables de se «différencier» à la suite de la formation de complexes moléculaires en réponse à un signal donné. Dès lors, la suppression de cavéoles pourrait entraîner la formation de structures de type radeaux, compensatrices. Ceci reste, bien entendu, à démontrer. II est vraisemblable que d'autres examens histologiques pratiqués sur ces souris ainsi que l'analyse de nouveaux tests devraient prochainement amener à préciser le rôle fonctionnel des cavéoles. Un point qui n'a pas été abordé dans ces études est le rôle présomptif des cavéoles dans l'homéostasie du cholestérol. Comment réagiraient ces souris si on les soumettait à un régime riche en cholestérol? Les souris sans cavéoles ont très certainement d'autres particularités à nous dévoiler. $\diamond$ Life without caveolae

1. Brown DA. Seeing is believing: Visualization of rafts in model membranes. Proc Natl Acad Sci USA 2001 ; 98: 10517-8.

2. Brown DA, Jacobson K. Microdomains, lipid rafts and caveolae (San Feliu de Guixols, Spain, 19- 24 May 2001). Traffic 2001 ; 2 : 668 72.

3. Drab M, Verkade P, Elger M, et al. Loss of caveolae, vascular dysfunction, and pulmonary defects in caveolin-1 gene-disrupted mice. Science 2001 ; 293: 2449-52.

4. Razani B, Engelman JA, Wang $X B$, et al. Caveolin-1 null mice are viable, but show evidence of hyper- proliferative and vascular abnormalities. J Biol Chem 2001; 16 : 16.

5. Wells WA. An organelle knockout. J Cell Biol 2001; 154 : 904-5. 